\title{
Oxygen Extraction Fraction and Stroke Risk in Patients with Carotid Stenosis or Occlusion: A Systematic Review and Meta-Analysis
}

\author{
A. Gupta, H. Baradaran, A.D. Schweitzer, H. Kamel, A. Pandya, D. Delgado, D. Wright, S. Hurtado-Rua, Y. Wang, and P.C. Sanelli
}

\begin{abstract}
BACKGROUND AND PURPOSE: Increased oxygen extraction fraction on PET has been considered a risk factor for stroke in patients with carotid stenosis or occlusion, though the strength of this association has recently been questioned. We performed a systematic review and meta-analysis to summarize the association between increased oxygen extraction fraction and ipsilateral stroke risk.
\end{abstract}

MATERIALS AND METHODS: A comprehensive literature search was performed. We included studies with baseline PET oxygen extraction fraction testing, ipsilateral stroke as the primary outcome, and at least 1 year of follow-up. A meta-analysis was performed by use of a random-effects model.

RESULTS: After screening 2158 studies, 7 studies with 430 total patients with mean 30 -month follow-up met inclusion criteria. We found that 6 of 7 studies were amenable to meta-analysis. Although 4 of the 6 studies independently did not reach statistical significance, meta-analysis revealed a significant positive relationship between abnormal oxygen extraction fraction and future ipsilateral stroke, with a pooled OR of $6.04(95 \% \mathrm{Cl}, 2.58-14.12)$. There was no statistically significant difference in OR in the subgroup analyses according to testing method or disease site.

CONCLUSIONS: Abnormal oxygen extraction fraction remains a powerful predictor of stroke in carotid stenosis or occlusion and is a valuable reference standard to compare and validate MR imaging-based measures of brain oxygen metabolism. However, there is a need for further evaluation of oxygen extraction fraction testing in patients with high-grade but asymptomatic carotid disease.

ABBREVIATIONS: $\quad$ CVR = cerebrovascular reserve; OEF = oxygen extraction fraction; COSS = Carotid Occlusion Surgery Study

C arotid atherosclerotic disease remains a significant cause of stroke, with extracranial carotid disease accounting for approximately $20 \%$ of all strokes. ${ }^{1}$ The hemodynamic risk factors underlying stroke in patients with carotid disease $\mathrm{e}^{2,3}$ include impairment in cerebrovascular reserve (CVR) and increase in oxy-

Received March 21, 2013; accepted after revision April 29.

From the Departments of Radiology (A.G., H.B., A.D.S., Y.W., P.C.S.) and Neurology (H.K.), Weill Cornell Medical College, New York-Presbyterian Hospital, New York, New York; and Department of Public Health (A.P., S.H.-R., P.C.S.) and Samuel J.

Wood Library and C.V. Starr Biomedical Information Center (D.D., D.W.), Weill Cornell Medical College, New York, New York.

Abstract previously presented at: Annual Meeting of the American Society of Neuroradiology, May 20-24, 2013; San Diego, California. The abstract for this paper won the Cornelieus G. Dyke Memorial Award at the meeting.

This paper was supported in part by NIH grant R01EB013443.

Please address correspondence to Ajay Gupta, MD, 525 East 68th St, Starr 8A, Box 141, New York, NY 10065; e-mail: ajg9004@med.cornell.edu

- Indicates open access to non-subscribers at www.ajnr.org

三 Indicates article with supplemental on-line tables

http://dx.doi.org/10.3174/ajnr.A3668 gen extraction fraction (OEF). Impairment in CVR, a measure of the vasodilatory capacity of vessels in the face of reduced cerebral perfusion pressure, may lead to a reduction in CBF, which can precede or occur alongside a compensatory increase in oxygen extraction state sometimes referred to as "misery perfusion." Neuroimaging can measure both cerebrovascular and oxygen metabolic reserve, with the latter determined by OEF on PET.

Increased OEF on PET has long been considered a risk factor for the development of stroke in patients with symptomatic carotid occlusion. ${ }^{4}$ However, OEF-defined hemodynamic failure was a key inclusion criterion for patients enrolled in the Carotid Occlusion Surgery Study (COSS). ${ }^{5}$ This trial was recently terminated for futility, and concerns were raised regarding the specific OEF testing method that was used as a study inclusion criterion, thus sparking renewed debate in the literature ${ }^{6-9}$ regarding OEF testing methodology and its role in stroke risk assessment. The role of OEF in stroke risk prediction also deserves renewed attention, given its potential value as a reference standard for new MR imaging measures of brain oxygen metabolism. ${ }^{10-12}$ Some of the 
difficulties in drawing definite conclusions about the role of OEF in predicting stroke and its role in treating patients with carotid disease are based on small sample sizes in individual research studies on this topic and the heterogeneity of study designs implemented. For this reason, and in the light of several recently published studies ${ }^{5,9}$ following patients after OEF PET testing, a critical reappraisal of the OEF literature is warranted. We therefore performed a systematic review and meta-analysis to summarize the association between increased OEF and risk of future stroke (first-ever or recurrent) ipsilateral to a high-grade carotid artery stenosis or occlusion.

\section{MATERIALS AND METHODS}

We referred to the Preferred Reporting Items for Systematic Reviews and Meta-Analyses (PRISMA) statement ${ }^{13}$ as a guide for the methodologic approach in this study.

\section{Study Eligibility Criteria}

Studies with PET-based measurement of OEF and its association with stroke in patients with high-grade carotid stenosis $(\geq 70 \%)$ or occlusion were eligible. Specific inclusion criteria were 1) English-language published manuscripts; 2) original prospective or retrospective research studies; 3 ) subjects with high-grade carotid stenosis $(\geq 70 \%)$ or occlusion determined by any imaging technique; 4) measurement of OEF by means of ${ }^{15} \mathrm{O}$-PET scan; 4) mean follow-up of $\geq 1$ year assessing development of ipsilateral stroke and/or TIA; and 5) nonsurgical treatment of patients. If surgical revascularization occurred during patient follow-up, we included the study only if the authors separately identified and analyzed these patients. In such a case, we included follow-up until the point of revascularization, at which time follow-up was censored. In cases in which outcome data or information about the OEF testing method could not be determined from the report, we attempted to contact the corresponding author for additional details. If 2 different testing methods were described in the original report, we applied the following rules: 1) the measure of OEF alone (eg, OEF alone instead of OEF plus additional hemodynamic parameters) was used in the overall analysis of pooled effect size; and 2) if 2 purely OEF-based testing methods were described in the same original report, the method that most accurately predicted stroke was used in the overall analysis of pooled effect size.

\section{Information Sources and Search}

A systematic search was performed to comprehensively identify studies predicting the risk of stroke or TIA on the basis of positive ${ }^{15} \mathrm{O}-\mathrm{PET}$ scans in patients with carotid stenosis or occlusion.

Potentially relevant articles were found by searching the biomedical electronic databases Ovid MEDLINE (1946 to October 2012), EMBASE (1974 to October 2012), and The Cochrane Library (updated October 2012). Relevant subject heading and free text terms were used. Published, unpublished, and ongoing trials were identified by search of ClinicalTrials.gov. Additional records were identified by use of the Related Citations feature in PubMed and the Cited Reference Search in Web of Science. To improve the retrieval of the relevant information and to ensure the methodologic quality of the literature search, there was an external peer review of the primary MEDLINE search. The primary search was conducted in MEDLINE by use of the terms exp PositronEmission Tomography/OR (positron adj2 emission adj2 tomograph\$).tw. OR (PET or PETCT\$one or PET CT\$1).tw. OR (Oxygen-15 or O-15).tw. AND (Oxygen adj3 (extract\$ or fraction or ratio or rate or metaboli\$ or consumption)).tw. OR (OEF or OER or CMRO2).tw. OR (cerebr\$ adj3 (metaboli\$ or autoregulat\$ or reserve or blood or flow or volume or resistance or pressure or hemodynamic\$ or vasomotor\$ or impair\$)).tw. OR (CBF or rCBF or CVR or CPP).tw. AND exp Carotid Stenosis/ OR (carotid adj3 (stenos\$ or ulcer\$ or plaque\$ or narrow\$ or obstruct\$ or occlus\$ or constrict\$)).tw. OR (steno\$ occlus\$ or stenoocclus\$).tw. OR exp Stroke/ OR Stroke\$.tw.OR cerebrovascular.tw. OR ((brain or vascular or lacunar or venous or cerebral or ischemic) adj2 (accident\$ or infarct\$ or event $\$$ or attack\$)).tw. OR (cva or cvas).tw.

\section{Study Selection and Data Collection Process}

All eligible reports were screened by a single reader on the basis of title and abstract for possible inclusion. These reports were reviewed in their entirety by 3 independent readers to determine final inclusion, with disagreements resolved by consensus. Qualitative and quantitative study data were extracted from selected studies by 2 independent readers by use of a predetermined data collection template. All disagreements were resolved by an independent third reader as a tie-breaker.

\section{Assessment of Risk of Bias in Studies}

On the basis of our literature search, no standardized tool exists to assess the risk of bias in observational time-to-event cohort studies. Therefore, we adapted bias assessment criteria used in a previously published meta-analysis ${ }^{14}$ of stroke risk on the basis of imaging findings. The following criteria were applied: 1) reference standard bias was assessed by noting whether observers were blinded to OEF results when stroke outcomes were determined; 2) confounding bias was assessed by noting whether potentially confounding co-existent vascular risk was collected and described; 3) completeness of follow-up data was determined by recording the number of subjects either censored or lost to follow-up for other reasons.

\section{Statistical Analyses}

A fixed-effects model was used if studies were found statistically homogeneous; otherwise, a random-effects model was chosen. The upper $95 \%$ confidence limit of the heterogeneity index $\left(I^{2}\right.$ $>30 \%$ ) was used as a cutoff for accepting studies that were relatively homogeneous. Heterogeneity across studies was also examined by means of the Breslow-Day method, with $P<.05$ as the threshold for statistically significant heterogeneity. Continuity correction was used for sparse tables before pooling the OR. Publication bias was examined with the use of Begg-Mazumdar tests. We performed subgroup analyses stratified by 1) disease site, 2) symptomatic disease versus never-symptomatic disease, and 3) absolute measures of OEF versus hemispheric ratio-based measures of OEF. All analyses were conducted by a biostatistician with the use of R: A Language and Environment for Statistical Computing, version 2.15.2 (http://www.r-project.org/).

AJNR Am J Neuroradiol 35:250-55 Feb 2014 www.ajnr.org 

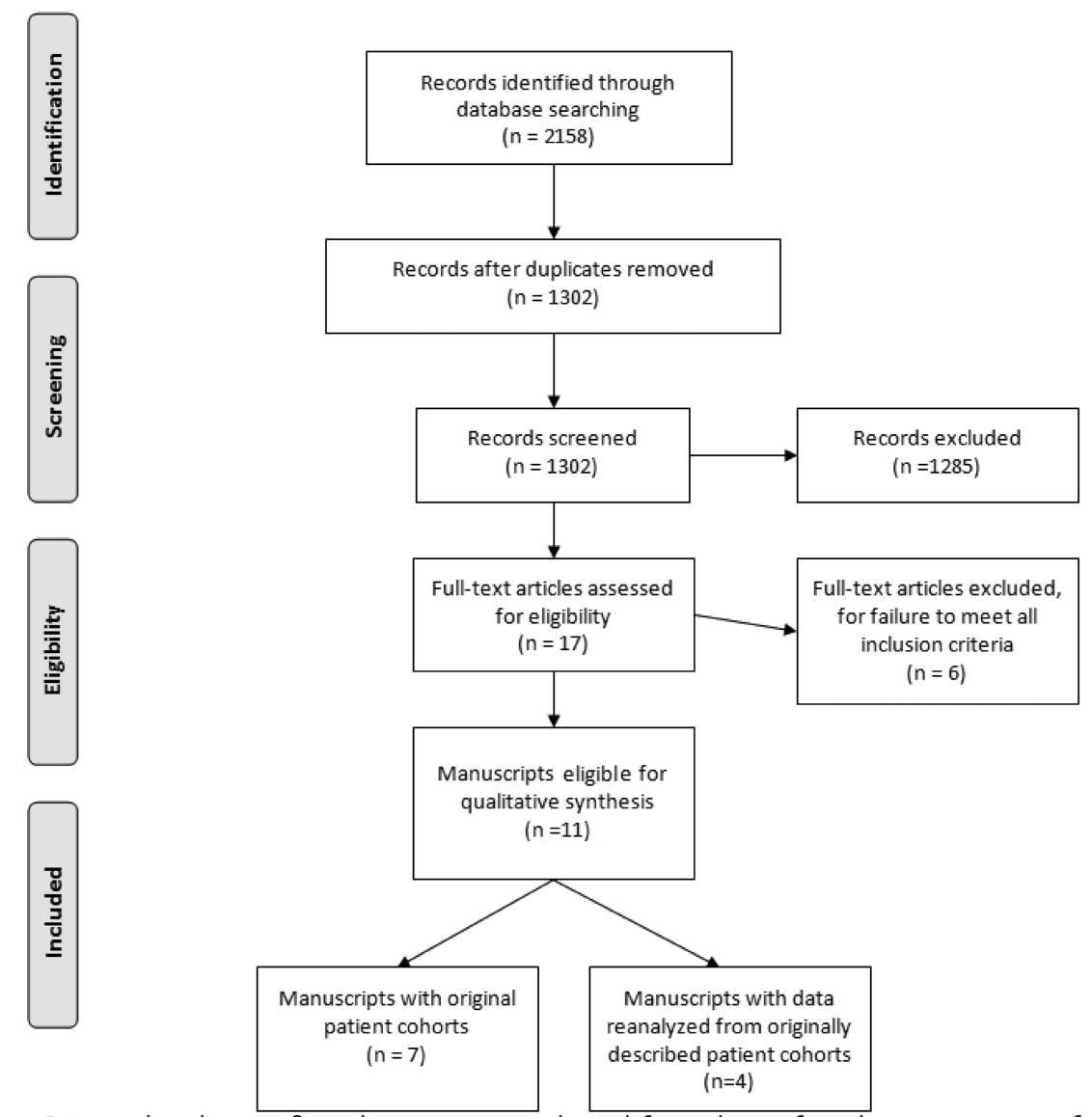

FIG 1. Study selection flow diagram. Figure adapted from the Preferred Reporting Items for Systematic Reviews and Meta-Analyses (PRISMA) group statement.

\section{RESULTS}

\section{Study Selection}

A total of 2158 reports were initially screened, of which 17 potentially eligible reports were selected for further review (Fig 1). Of these 17 reports, 6 did not meet inclusion criteria when read in their entirety because they did not include patient cohorts followed for development of stroke after baseline OEF testing. Of the remaining 11 reports, 7 reports ${ }^{4,5,9,15-18}$ were included in the final systematic review. The remaining 4 of 11 reports ${ }^{3,8,19,20}$ reanalyzed data originally presented in an original patient cohort. ${ }^{4}$ Two ${ }^{9,16}$ of 7 of these original cohort studies described more than 1 OEF testing method for the cohort, whereas the other 5 described only 1 method of OEF testing. Of the 7 original cohort studies for systematic review, 6 of 7 studies reported ipsilateral stroke incidence in a fashion amenable to meta-analysis. One study (the Carotid Occlusion Surgery Study ${ }^{5}$ ) reported stroke outcomes only in patients with abnormal OEF, which prevented calculation of an OR for inclusion in the meta-analysis. The stroke outcomes data for patients with normal OEF who were excluded from the randomized trial could not be obtained after contacting the study authors.

\section{Qualitative Assessment and Study Characteristics}

Of the 7 original cohort reports meeting eligibility for qualitative review, 6 were nonrandomized, observational, time-to-event studies, and 1 was observational data extracted from a randomized, controlled trial. ${ }^{5}$ Four studies were conducted in the United
States ${ }^{4,5,15,17}$ and the remaining 3 studies in Japan. ${ }^{9,16,18}$ A total of 430 unique patients were included, with a mean follow-up of 30 months. All studies had similar mean subject ages (range, 58-66 years) and a similar increased preponderance of male subjects (range, 62$77 \%)$. Most patients enrolled in these studies had occlusive ICA disease, with 3 studies exclusively studying this population., ${ }^{4,17}$ The remaining 4 studies ${ }^{9,15,16,18}$ had mixed disease sites and severity, including high-grade stenosis and/or occlusion of the middle cerebral and carotid arteries. In studies with mixed vessel site and severity, most patients had occlusive as opposed to stenotic vascular disease (comprising $83.3 \%,{ }^{15} 80 \%,{ }^{16} 75 \%,{ }^{18}$ and $67.3 \%{ }^{9}$ of each of the cohorts); however, stroke outcomes were not consistently reported in all of these studies by vessel disease site or severity. All but 1 study ${ }^{17}$ evaluated patients with symptomatic disease, which was defined by the presence of prior TIA or stroke with variable days since last symptoms. Online Table 1 provides an overview of the patient characteristics in each study.

Variable cutoff values for abnormal OEF were used, with major testing categories including 1) quantitative, arterial catheterization-dependent versus non-arterial-dependent count-based OEF techniques and 2) absolute versus hemispheric ratio OEF techniques (On-line Table 2). All studies presented outcomes in terms of ipsilateral stroke. Original cohort data from Grubb et $\mathrm{al}^{4}$ were reanalyzed 6 times in 4 subsequent reports ${ }^{3,7,19}$ (On-line Tables $3-5)$. In 2 reports by Yamauchi et al, ${ }^{9,16}$ more than 1 OEF testing method was presented.

\section{Assessment of Study Methods}

In only 2 observational cohort studies ${ }^{4,17}$ were researchers explicitly blinded to OEF results when assessing for ipsilateral stroke. In $4^{9,15,16,18}$ of the remaining 5 studies, outcomes were not assessed while blinded to OEF results, and in the Carotid Occlusion Surgery Study participants were selected on the basis of known preexisting OEF elevation. All 7 studies measured and described potentially confounding pre-existing vascular factors. Finally, in the assessment of the completeness of follow-up, the COSS trial ${ }^{5}$ lost 1 patient to follow-up at 21 months. In the other 6 studies, no explicit loss to follow-up was described.

\section{Meta-Analysis Results}

After pooling the 6 studies amenable for meta-analysis, the $I^{2}$ statistic and Breslow-Day statistic showed low heterogeneity $\left(I^{2}=\right.$ 0 ; $\mathrm{CI}=0-38.3 \%$ and Breslow-Day $=2.39, d f=5, P=.66)$. The Begg-Mazumdar test did not reveal significant publication bias 


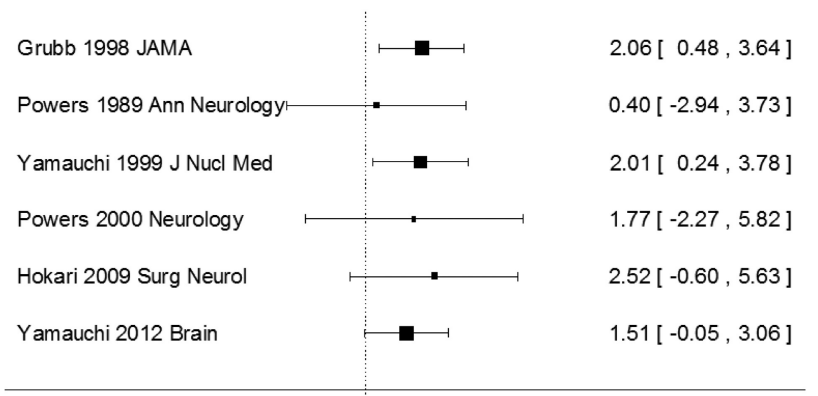

RE Model

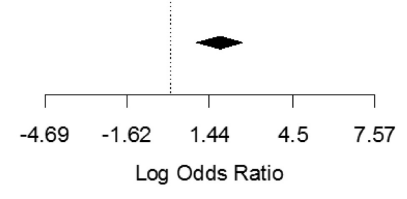

$1.80[0.95,2.65]$

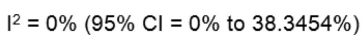

Breslow Day $=2.3907, p=0.6643$

FIG 2. Forest plot of the association between increased OEF and ipsilateral first-ever or recurrent stroke determined by a random effects (RE) model. Squares represent point estimates for effect size expressed as log of the $O R$, with the size proportional to the inverse variance of the estimate (note, $O R=\exp [\log O R])$. Diamonds represent pooled estimate. Lines represent $95 \% \mathrm{Cls}$. Vertical line indicates null effect $(\mathrm{OR}=1$ or log odds $=0) . I^{2}$ and Breslow-Day statistic for heterogeneity is listed below the forest plot.

(Kendall $\tau=.067, P=1.00)$. The pooled random effects odds ratio of 6.04 (CI, 2.58-14.12) indicates a significant positive relationship between increased OEF and future ipsilateral stroke (Fig 2). Each study had a positive association between OEF increase and stroke, though importantly, 75\% of the studies (4/6), when viewed independently, did not have a statistically significant OR.

\section{Subset Analysis}

Additional subset analyses with measures of heterogeneity were performed. The OR remained statistically significant in all of the subset analyses: 1) symptomatic patients only (Fig 3A) (only 1 study, Powers et $\mathrm{al}^{17}$ from 2000 studied asymptomatic patients and was not alone statistically significant), 2) disease site involving only the carotid artery (Fig $3 B$ ) versus patients with carotid and MCA disease (Fig 3C), and 3) testing by use of absolute OEF measures (Fig $4 A$ ) versus testing by use of OEF hemispheric ratiobased measures (Fig 4B).

\section{DISCUSSION}

In patients with carotid artery stenosis or occlusion, accurate measures of stroke risk are important for guiding management and treatment decisions. Though structural neuroimaging can play a role in measuring the degree of vessel narrowing, imaging of downstream hemodynamic factors can provide additional insight into stroke risk, including impairments in CVR. ${ }^{14}$ Often considered to be the end-stage of hemodynamic failure, increases in OEF as measured on PET were recently used to select patients for the COSS trial, randomly assigning subjects to surgical vascular bypass or medical therapy. With the recent early termination of this trial, there has been renewed interest in the predictive value of OEF increase because some authors ${ }^{6}$ have attributed the trial's futility to methodologic failure of the OEF PET selection criteria. In addition, with multiple MR imaging-based surrogate mea- sures of OEF being developed with techniques such as susceptibility-weighted MR imaging, ${ }^{10}$ functional MR imaging, ${ }^{11}$ and MR spectroscopy, ${ }^{12}$ it is important to summarize the value of OEF as a reference standard to predict stroke by which future technologies can be assessed. If shown to be accurate markers of cerebral oxygen metabolism compared with PET-derived OEF, these potentially more widely available MR imaging techniques can then be tested as potential stroke risk prediction tools.

In our systematic review and meta-analysis of 430 patients with a mean follow-up of 30 months, increased OEF was strongly associated with the risk of ipsilateral stroke, though when viewed independently, only 2 of the 6 studies included in the meta-analysis reached statistical significance. The pooled OR suggests that despite variability in results of individual studies in the literature, patients with increased OEF are approximately 6 times more likely to have ipsilateral stroke than those without increased OEF. Most patients in this study had symptomatic occlusive arterial disease; only 1 study ${ }^{17}$ contained a cohort of never-symptomatic patients. This study did not find a significant association between increased OEF and stroke but was limited by its small sample size. The need for stroke prediction in patients with carotid occlusion, the disease state in which most OEF investigation has been done, appears questionable, given the results of COSS. However, the strong performance of OEF in predicting stroke in this population suggests that it may also be useful in patients with high-grade but asymptomatic carotid stenosis, for whom accurate stroke prediction is likely to prove important in deciding between intervention and medical therapy. ${ }^{21}$ Our study emphasizes the need for prospective investigation evaluating the role of OEF testing in predicting stroke risk in asymptomatic carotid stenosis, especially in light of data that impairment of CVR has been associated with stroke in such patients. ${ }^{14}$

We also found no significant difference in broad categories of OEF testing method and stroke risk prediction. Specifically, we did not find a significant difference in OR when absolute values of OEF were calculated versus hemispheric-ratio-based techniques (On-line Table 2). This is of note because Carlson et $\mathrm{al}^{6}$ recently commented that semiquantitative hemispheric OEF ratios for patient selection in COSS probably contributed to trial failure, which suggests that this method is inferior to quantitative, absolute measures of OEF. Although our study was not designed specifically to analyze this issue, we found insufficient evidence to claim superiority of one of these methods over the other. Even in a study by Yamauchi et al, ${ }^{16}$ in which absolute OEF testing performed better than ratio-based techniques in predicting stroke in the same patients, this difference was not statistically significant. As far as optimizing thresholds for classification of abnormal OEF test results, it is interesting to note that studies that had repeated analyses of patient data suggest that adding measures of cerebral blood volume and CBF to OEF may help to further define higher hemodynamic risk categories. ${ }^{3,9}$

Our study has some limitations. Although the outcome measure (ipsilateral stroke) was the same across studies, the exact definition of stroke was not uniform, given the variable criteria for each study, and in only 2 of the 6 included studies were adjudication of stroke outcomes made blinded to OEF data. Similarly, stroke outcomes were not consistently reported broken down by 


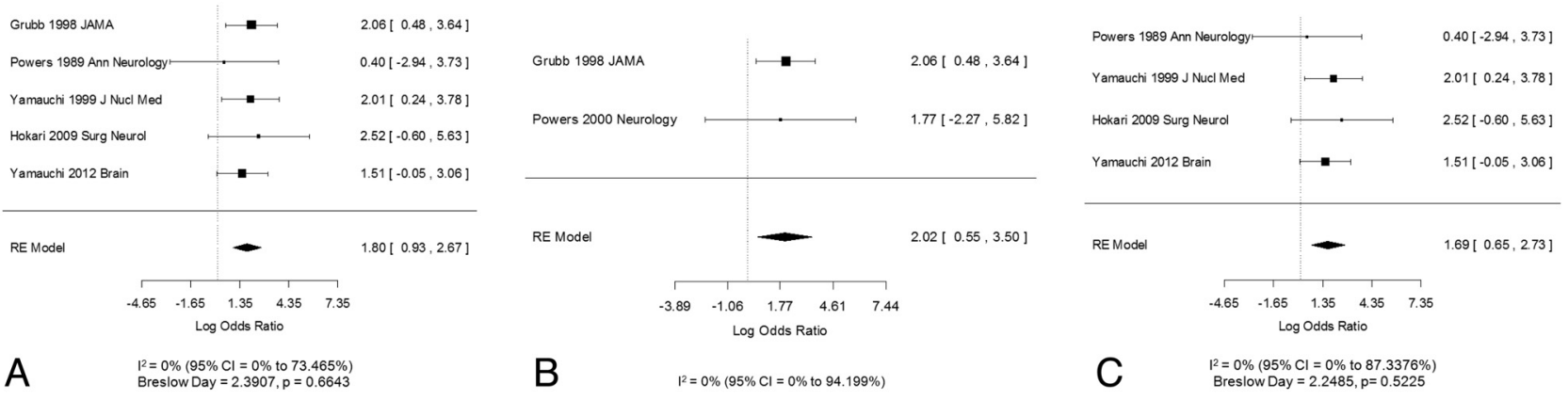

FIG 3. A, Symptomatic patients only, with the OR representing the strength of association between increased OEF and risk of either recurrent stroke or stroke preceded by prior TIA in the symptomatic territory. B, Carotid artery disease only. C, Carotid and MCA disease. Log OR and 95\% $\mathrm{Cl}$ for studies divided by the presence of disease in symptomatic patients only $(A)$, in the carotid artery only $(B)$, or in the carotid artery and MCA (C). Note that a Breslow-Day statistic could not be calculated for the forest plot in $B$, given the small outcome events. Squares represent point estimates for effect size expressed as log of the OR, with the size proportional to the inverse variance of the estimate (note, OR = exp [log OR]). Diamonds represent pooled estimate. Lines represent $95 \% \mathrm{Cls}$. Vertical line indicates null effect $(\mathrm{OR}=1$ or log odds $=0) . I^{2}$ and Breslow-Day statistic for heterogeneity is listed below the forest plot.

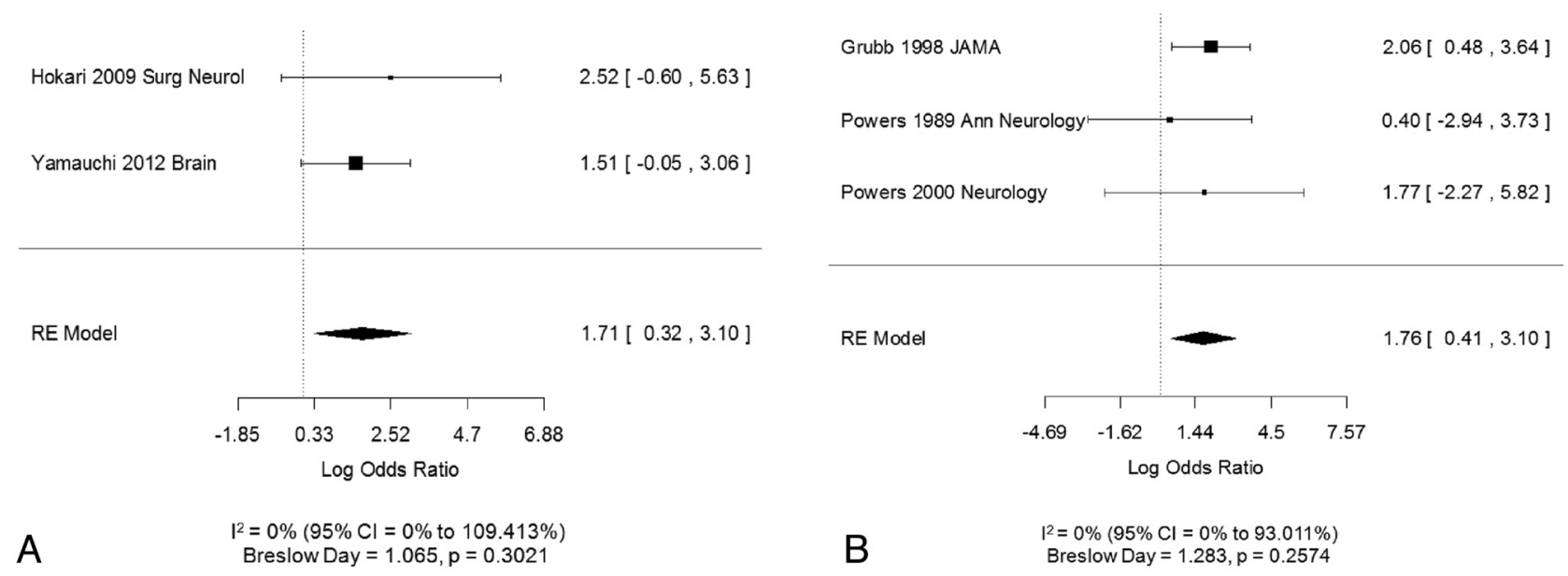

FIG 4. A, Testing by use of absolute OEF measures. $B$, Testing by use of hemispheric ratio-based measures. Log OR and $95 \% \mathrm{Cl}$ for studies divided by the OEF testing by use of absolute measures of OEF $(A)$ or hemispheric ratio-based measure of OEF (B). Squares represent point estimates for effect size expressed as log of the OR, with the size proportional to the inverse variance of the estimate (note, OR = exp [log OR]). Diamonds represent pooled estimate. Lines represent $95 \% \mathrm{Cls}$. Vertical line indicates null effect (OR $=1$ or log odds $=0) . I^{2}$ and Breslow-Day statistic for heterogeneity is listed below the forest plot.

vessel disease site or severity in those studies with mixed patient characteristics thereby preventing more detailed subset analyses in these studies. In addition, though most studies focused on occlusive disease, in those cohorts in which stenosis was present, individual patient-level results are confounded, in part, by the fact that groups with and without OEF elevation did not have exact matching degrees of arterial stenosis. In addition, as the withincohort subset analyses demonstrate, definitions of abnormal OEF can vary and significantly affect the resulting OR. Unfortunately, the data were not amenable to comparisons of receiver operatingcharacteristic curves, a potentially more useful measure of diagnostic test performance. Beyond this, comparing imaging test data across different PET scanners and techniques prevents deriving specific cutoffs for defining abnormal OEF that could be broadly applied to various institutions. Finally, these studies all analyzed a hemodynamic risk factor underlying stroke and did not differentiate strokes that may have arisen from embolic phenomena, potentially overstating the strength of the association between OEF elevation and stroke.

Despite these limitations, our study suggests that increased
OEF remains a robust predictor of ipsilateral stroke in patients with symptomatic carotid disease across multiple disease sites and across broad categories of testing methodology. Furthermore, our study has shown that oxygen metabolism and stroke risk in asymptomatic carotid stenosis requires further study, given the paucity of OEF literature in this patient population in which the identifying high-risk subgroups could be of significant value. Despite the challenges of routinely implementing ${ }^{15} \mathrm{O}$-PET scanning, our study supports the use of testing as a marker of stroke risk and emphasizes the need for continued investigation of newer techniques to measure cerebral oxygen metabolism.

\section{ACKNOWLEDGMENT}

The Association of University Radiologists General Electric Radiology Research Academic Fellowship (GERRAF) is acknowledged for supporting a portion of Dr Gupta's efforts.

Disclosures: Ajay Gupta_RELATED: Grant: AUR-GE Radiology Research Academic Fellowship, ${ }^{*}$ Comments: Part of Dr Gupta's effort for this study was supported by a AUR-GERRAF award from 2012-2014. Yi Wang-RELATED: Grant: NIH R01EB013443*; 
UNRELATED: Patents (planned, pending or issued): One of the inventors on QSM patent application. * Pina Sanelli-RELATED: Grant: NIH NINDS* ( ${ }^{*}$ money paid to institution).

\section{REFERENCES}

1. Petty GW, Brown RD Jr, Whisnant JP, et al. Ischemic stroke subtypes: a population-based study of incidence and risk factors. Stroke 1999;30:2513-16

2. Silvestrini M, Vernieri F, Pasqualetti P, et al. Impaired cerebral vasoreactivity and risk of stroke in patients with asymptomatic carotid artery stenosis. JAMA 2000;283:2122-27

3. Derdeyn CP, Videen TO, Yundt KD, et al. Variability of cerebral blood volume and oxygen extraction: stages of cerebral haemodynamic impairment revisited. Brain 2002;125:595-607

4. Grubb RL Jr, Derdeyn CP, Fritsch SM, et al. Importance of hemodynamic factors in the prognosis of symptomatic carotid occlusion. JAMA 1998;280:1055-60

5. Powers WJ, Clarke WR, Grubb RL Jr, et al. Extracranial-intracranial bypass surgery for stroke prevention in hemodynamic cerebral ischemia: the Carotid Occlusion Surgery Study randomized trial. JAMA 2011;306:1983-92

6. Carlson AP, Yonas H, Chang YF, et al. Failure of cerebral hemodynamic selection in general or of specific positron emission tomography methodology? Carotid Occlusion Surgery Study (COSS). Stroke 2011;42:3637-39

7. Powers WJ, Clarke WR, Adams HP Jr, et al. Commentary: extracranial-intracranial bypass for stroke in 2012: response to the critique of the carotid occlusion surgery study "it was deja vu all over again.” Neurosurgery 2012;71:E772-76

8. Powers WJ. Letter by Powers regarding article, "Failure of cerebral hemodynamic selection in general or of specific positron emission tomography methodology? Carotid Occlusion Surgery Study (COSS)." Stroke 2012;43:e43

9. Yamauchi H, Higashi $\mathrm{T}$, Kagawa $\mathrm{S}$, et al. Is misery perfusion still a predictor of stroke in symptomatic major cerebral artery disease? Brain 2012;135:2515-26

10. Zaitsu Y, Kudo K, Terae S, et al. Mapping of cerebral oxygen extrac- tion fraction changes with susceptibility-weighted phase imaging. Radiology 2011;261:930-36

11. Gauthier CJ, Desjardins-Crepeau L, Madjar C, et al. Absolute quantification of resting oxygen metabolism and metabolic reactivity during functional activation using QUO2 MRI. Neuroimage 2012;63:1353-63

12. Zhu XH, Chen JM, Tu TW, et al. Simultaneous and noninvasive imaging of cerebral oxygen metabolic rate, blood flow and oxygen extraction fraction in stroke mice. Neuroimage 2013;64:437-47

13. Liberati A, Altman DG, Tetzlaff J, et al. The PRISMA statement for reporting systematic reviews and meta-analyses of studies that evaluate health care interventions: explanation and elaboration. Ann Intern Med 2009;151:W65-94

14. Gupta A, Chazen JL, Hartman M, et al. Cerebrovascular reserve and stroke risk in patients with carotid stenosis or occlusion: a systematic review and meta-analysis. Stroke 2012;43:2884-91

15. Powers WJ, Tempel LW, Grubb RL Jr. Influence of cerebral hemodynamics on stroke risk: one-year follow-up of 30 medically treated patients. Ann Neurol 1989;25:325-30

16. Yamauchi H, Fukuyama H, Nagahama Y, et al. Significance of increased oxygen extraction fraction in five-year prognosis of major cerebral arterial occlusive diseases. J Nucl Med 1999;40:1992-98

17. Powers WJ, Derdeyn CP, Fritsch SM, et al. Benign prognosis of never-symptomatic carotid occlusion. Neurology 2000;54:878-82

18. Hokari M, Kuroda S, Shiga T, et al. Impact of oxygen extraction fraction on long-term prognosis in patients with reduced blood flow and vasoreactivity because of occlusive carotid artery disease. Surg Neurol 2009;71:532-8

19. Derdeyn CP, Videen TO, Simmons NR, et al. Count-based PET method for predicting ischemic stroke in patients with symptomatic carotid arterial occlusion. Radiology 1999;212:499-506

20. Derdeyn CP, Videen TO, Grubb RL Jr, et al. Comparison of PET oxygen extraction fraction methods for the prediction of stroke risk. J Nucl Med 2001;42:1195-97

21. Abbott AL. Medical (nonsurgical) intervention alone is now best for prevention of stroke associated with asymptomatic severe carotid stenosis: results of a systematic review and analysis. Stroke 2009;40: e573-83 